\title{
ON SEMI-OPEN SETS AND \\ MUTUAL CORRESPONDENCE BETWEEN \\ PROPERTIES OF FUNCTIONS CONSIDERED WITH RESPECT \\ TO DIFFERENT TOPOLOGICAL STRUCTURES
}

\author{
Ewa Korczak-Kubiak - Ryszard J. Pawlak
}

\begin{abstract}
At the end of the twentieth century, Á. Császár pointed out the possibility of the transition from topology to generalized topology and vice versa. One of the tools used for this purpose are semi-open sets. In this paper, we investigate properties of functions in GTS in the context of semi-open sets, and problems connected with transfer of properties of functions defined and assuming their values in primal spaces to functions considered as mappings in the spaces generated by primal spaces.
\end{abstract}

\section{Introduction and preliminaries}

In [7, Á. C sás zár introduced the notion of generalized topological space (GTS). The issues connected with these spaces became the basis of many scientitic papers, e.g., [3], 88, [10], [12], [17, [19], [25]. In [9], generalized topological spaces were related, among others, with semi-open sets. It turned out that it leads to building new GTSs, because the family of all semi-open sets forms a generalized topology. In this way, if we use a topological space as our primal space then, by considering the family of all semi-open sets, we will obtain a generalized topology and a generalized topological space generated by the primal space. On the other hand, if we have strong generalized topological space, then the family of all sets, which intersection with any generalized open set is a generalized open set, forms a topology. Of course, in extreme cases, the topology consists only of an empty set and the whole space. So, we have a transition in opposite side: from sGTS to topological space.

(C) 2016 Mathematical Institute, Slovak Academy of Sciences.

2010 Mathematics Subject Classification: 54A05, 54C08, 26A15, 54H20.

Keyw ords: semi-open set, generalized topological space, Baire generalized topological space, transitive function, nomadic function. 
In this paper, we will present results connected with a few issues. First of all, semi-open sets will be used as a tool to analyse some Baire properties of GTS. In the second part of the paper, we will deal with various kinds of transitivity (weakly nomadic, nomadic and transitive functions). Many studies connected with the problems of transitivity was aimed at determining some conditions which implies the equivalence of transitivity and nomadic property. Our research differs from the earlier results because, in one issue, we combine the problem of equivalence of various kinds of transitivity and the problem of transition from one topological structure to the second one.

Why are these concepts so important? The answer seems to be simple. They are closely connected with many interpretations of chaos, e.g., Li-Yorke chaos, Devaney chaos, Auslander-Yorke chaos, etc.

We will mostly use standard notations. In particular, letters $\mathbb{R}, \mathbb{Q}$ and $\mathbb{N}$ will denote the sets of all real numbers, rational numbers and positive integers, respectively. The symbol $\mathcal{T}_{\mathbb{Q}}$ will stand for the induced topology on $\mathbb{Q}$. Moreover, for any nonempty set $X$, we will use the symbol $\exp (X)$ to denote the family of all subsets of $X$.

In this paper, we will consider, among others, generalized topological spaces. Let $X \neq \emptyset$. We say that a family $\gamma \subset \exp (X)$ is a generalized topology (GT, for short) in $X$ if $\emptyset \in \gamma$ and $\bigcup_{t \in T} G_{t} \in \gamma$ whenever $\left\{G_{t}: t \in T\right\} \subset \gamma$. A pair $(X, \gamma)$ will be called a generalized topological space (GTS, for short). If $X \in \gamma$, then we say that $(X, \gamma)$ is a strong generalized topological space (sGTS, for short).

We will write $\operatorname{int}_{\gamma}(A)$ to denote an interior of a set $A$ in generalized topology $\gamma$, and $\operatorname{cl}_{\gamma}(A)$ will denote a closure of a set $A$ in $\gamma$.

It is worth noting that in GTS an interior and a closure of a set are defined in the same way as in a topological space. However, they may have different properties. For example, throughout the paper, we will use the following fact: $\mathrm{cl}_{\gamma}(\emptyset)=\emptyset$ if and only if $(X, \gamma)$ is sGTS.

We say that a set $A \subset X$ is semi-open if $A \subset \operatorname{cl}_{\gamma}\left(\operatorname{int}_{\gamma}(A)\right)$. The family of all semi-open sets with respect to $\gamma$ will be denoted by $\sigma(\gamma)$, i.e., $\sigma(\gamma)=\{A \subset X$ : $\left.A \subset \operatorname{cl}_{\gamma}\left(\operatorname{int}_{\gamma}(A)\right)\right\}$. Space $(X, \sigma(\gamma))$ is sGTS ([7]). As in the case of a topological space, if $(X, \gamma)$ is GTS, then we say that $A \subset X$ is $\gamma$-dense if $\operatorname{cl}_{\gamma}(A)=X$ or equivalently $A \cap U \neq \emptyset$ for any $U \in \gamma \backslash\{\emptyset\}$. We will omit the prefix $\gamma$ when no confusion can arise.

Let $\mathcal{P}$ be a family of subsets of a fixed nonempty set $X$ such that $\emptyset \in \mathcal{P}$. By $\gamma_{\mathcal{P}}$ we will denote a family of all possible unions of the sets from $\mathcal{P}$. It is easy to see that $\gamma_{\mathcal{P}}$ is a generalized topology.

The issue of functions in the context of semi-open sets was considered in many papers, e.g., [1], 4], 6], 11], [14, [23. Since the terminology within the scope 


\section{ON SEMI-OPEN SETS OF FUNCTIONS}

of this issue is not unified, we will present definitions of basic kinds of functions connected with semi-open sets.

Let $\left(X, \gamma_{X}\right)$ and $\left(Y, \gamma_{Y}\right)$ be GTSs. A function $f:\left(X, \gamma_{X}\right) \rightarrow\left(Y, \gamma_{Y}\right)$ is called:

- semi-continuous if for each $V \in \gamma_{Y}$ the set $f^{-1}(V)$ is semi-open in $\left(X, \gamma_{X}\right)$;

- irresolute if for each set $V$ semi-open in $\left(Y, \gamma_{Y}\right)$ the set $f^{-1}(V)$ is semi-open in $\left(X, \gamma_{X}\right)$;

- pre-semi-open if for each set $V$ semi-open in $\left(X, \gamma_{X}\right)$ the set $f(V)$ is semi-open in $\left(Y, \gamma_{Y}\right)$;

- semi-open if for each set $V \in \gamma_{X}$ the set $f(V)$ is semi-open in $\left(Y, \gamma_{Y}\right)$.

We say that a surjection $f:\left(X, \gamma_{X}\right) \rightarrow\left(Y, \gamma_{Y}\right)$ is:

- a $q$-homemorphism if $f$ is continuous and open;

- a q-semi-homeomorphism if $f$ is semi-continuous and semi-open.

Since the issues connected with transitivity of functions will also be considered in this paper, let us adopt the classical notations used in discrete dynamical systems. If $f: X \rightarrow X$ then $f^{0}(x)=x$ and $f^{i}(x)=f\left(f^{i-1}(x)\right)$, for $i \in \mathbb{N}$. If $A \subset X$ then $f^{-k}(A)=\left\{x \in X: f^{k}(x) \in A\right\}$ for $k \in \mathbb{N} \cup\{0\}$. An orbit of a point $x$ with respect to a function $f$ (denoted by $\left.\Theta_{f}(x)\right)$ is a set (sequence) $\left\{x, f(x), f^{2}(x), \ldots\right\}$.

\section{Baire spaces and semi-open sets}

Let $(X, \gamma)$ be GTS. We say that a set $A \subset X$ is $\gamma$-nowhere dense if $\operatorname{int}_{\gamma}\left(\operatorname{cl}_{\gamma}(A)\right)=\emptyset$. A set $A \subset X$ is $\gamma$-strongly nowhere dense if for any nonempty set $V \in \gamma$ there exists a nonempty set $U \in \gamma$ such that $U \subset V$ and $A \cap U=\emptyset$. It should be mentioned here that the union of two $\gamma$-nowhere dense sets need not be $\gamma$-nowhere dense while the union of two $\gamma$-strongly nowhere dense sets is $\gamma$-strongly nowhere dense $([18])$.

We shall say that $A$ is a $\gamma$-meager ( $\gamma$-s-meager) set if $A=\bigcup_{n=1}^{\infty} A_{n}$ and $A_{n}$ are $\gamma$-nowhere dense ( $\gamma$-strongly nowhere dense) for all $n \in \mathbb{N}$. We shall say that $A$ is a $\gamma$-II-category set ( $\gamma$-s-II category set) if $A$ is not a $\gamma$-meager ( $\gamma$-s-meager) set.

Using the above notions, in the further part of the paper, we will omit prefix $\gamma$ when no confusion can arise.

We shall say that $(X, \gamma)$ is a weak Baire space, wBS for short (Baire space, BS for short) if each nonempty set $V \in \gamma$ is of s-II category (II category).

LEMMA 2.1. GTS $(X, \gamma)$ is a Baire space if and only if each countable union of closed and nowhere dense subsets of $X$ has an empty interior. 
P r o o f. Necessity is obvious.

Sufficiency. Suppose that $(X, \gamma)$ is not a Baire space. Then, there exists a nonempty meager set $U \in \gamma$. Thus $U=\bigcup_{n \in \mathbb{N}} K_{n}$, where $K_{n}$ is nowhere dense for each $n \in \mathbb{N}$. Clearly, $U \subset \bigcup_{n \in \mathbb{N}} \operatorname{cl}\left(K_{n}\right)$ and hence

$$
\operatorname{int}\left(\bigcup_{n \in \mathbb{N}} \operatorname{cl}\left(K_{n}\right)\right) \neq \emptyset \text {. }
$$

By Property 2.3 [18], we have that $\operatorname{cl}\left(K_{n}\right)$ is a nowhere dense set for each $n \in \mathbb{N}$. Thus, the set $\bigcup_{n \in \mathbb{N}} \operatorname{cl}\left(K_{n}\right)$ is a countable union of closed and nowhere dense sets, and so it has an empty interior. This contradicts (1).

In the case of topological spaces, it is well-known that a space is Baire if and only if each intersection of sequence consisting of open and dense sets is a dense set. In the case of GTS, the necessity of this condition has been only proved. The following theorem shows that a full generalization of this statement is true.

THEOREM 2.2. Let $(X, \gamma)$ be GTS. $(X, \gamma)$ is a Baire space if and only if each countable intersection of open and dense subsets of $X$ is dense.

P r o o f. Necessity is a consequence of Property 2 from [20].

Sufficiency. Let $\left\{F_{n}\right\}_{n \in \mathbb{N}}$ be an arbitrary sequence of closed and nowhere dense sets. Due to Lemma 2.1] it suffices to show that

$$
F=\bigcup_{n \in \mathbb{N}} F_{n} \text { has an empty interior. }
$$

First, we will prove that

$$
X \backslash F_{n} \text { is an open and dense set, for each } n \in \mathbb{N} \text {. }
$$

Obviously, we have $X \backslash F_{n} \in \gamma$ for each $n \in \mathbb{N}$.

Now, we will show that $X \backslash F_{n}$ is a dense set for each $n \in \mathbb{N}$. Suppose, contrary to our claim, that there exists $n_{0} \in \mathbb{N}$ such that $X \backslash F_{n_{0}}$ is not a dense set. Then, there exists a nonempty set $U \in \gamma$ such that $U \cap\left(X \backslash F_{n_{0}}\right)=\emptyset$. Hence $U \subset F_{n_{0}} \subset \operatorname{cl}\left(F_{n_{0}}\right)$, so $\operatorname{int}\left(\operatorname{cl}\left(F_{n_{0}}\right)\right) \neq \emptyset$, which is impossible because $F_{n_{0}}$ is nowhere dense. The proof of (2) is finished.

By the assumption, $X \backslash F=\bigcap_{n \in \mathbb{N}}\left(X \backslash F_{n}\right)$ is dense. Consequently, $\operatorname{int}(F)=\emptyset$.

Our next considerations will regard the problem of preserving Baire property of sGTS under mappings. We will start with showing that for each Baire sGTS $X$ a countable sum of $X$ is Baire sGTS such that some continuous image of this space is not Baire GTS. First, let us introduce the necessary definitions.

Analogously to the notion of a sum of pairwise disjoint topological spaces ([13]), we can consider a countable sum of pairwise disjoint sGTSs. Let $(X, \gamma)$ 
be sGTS. For $n \in \mathbb{N}$, consider $\left(X_{n}, \gamma_{n}\right)$, where $X_{n}=X \times\{n\}$ and $\gamma_{n}=\{A \times\{n\}$ : $A \in \gamma\}$. Obviously, $\left(X_{n}, \gamma_{n}\right)$ is sGTS for $n \in \mathbb{N}$. Then, $\oplus_{n \in \mathbb{N}}\left(X_{n}, \gamma_{n}\right)$ is sGTS and $\oplus_{n \in \mathbb{N}}\left(X_{n}, \gamma_{n}\right)=\left(Y, \gamma_{\mathcal{P}}\right)$, where $Y=X \times \mathbb{N}$ and $\mathcal{P}=\{A \times\{n\}: A \in \gamma \wedge n \in \mathbb{N}\}$. In the further part of the paper, we use symbol $\oplus(X, \gamma)$ to denote $\oplus_{n \in \mathbb{N}}\left(X_{n}, \gamma_{n}\right)$.

Theorem 2.3. Let $(X, \gamma)$ be a Baire sGTS. Then, $\oplus(X, \gamma)$ is a Baire sGTS which can be mapped by a continuous function onto the space $\left(\mathbb{Q}, \mathcal{T}_{\mathbb{Q}}\right)$.

P r o o f. Let $(X, \gamma)$ be a Baire sGTS. Adopt the following notation: if $A \subset X \times \mathbb{N}$, then $A_{n}=A \cap(X \times\{n\})$ for $n \in \mathbb{N}$. Obviously, $A_{n} \subset X \times\{n\}(n \in \mathbb{N})$. Put $\tilde{A}_{n}=\left\{x \in X:(x, n) \in A_{n}\right\}$. So, $A_{n}=\tilde{A}_{n} \times\{n\}$, for each $n \in \mathbb{N}$. It is easy to see that

$$
A \in \gamma_{\mathcal{P}} \text { if and only if } \tilde{A}_{n} \in \gamma, \quad \text { for } n \in \mathbb{N} .
$$

Notice first that

if there exists $n_{0} \in \mathbb{N}$ such that $A \subset X \times\left\{n_{0}\right\}$ then

$$
\operatorname{int}_{\gamma_{\mathcal{P}}}(A)=\operatorname{int}_{\gamma}\left(\tilde{A}_{n_{0}}\right) \times\left\{n_{0}\right\} \text { and } \operatorname{cl}_{\gamma_{\mathcal{P}}}(A)=\operatorname{cl}_{\gamma}\left(\tilde{A}_{n_{0}}\right) \times\left\{n_{0}\right\} .
$$

Now, we will prove that

if there exists $n_{0} \in \mathbb{N}$ such that $A \subset X \times\left\{n_{0}\right\}$ then

$A$ is nowhere dense in $\left(X \times \mathbb{N}, \gamma_{\mathcal{P}}\right)$ if and only if

$\tilde{A}_{n_{0}}$ is nowhere dense in $(X, \gamma)$.

Let $A \subset X \times\left\{n_{0}\right\}$ for some $n_{0} \in \mathbb{N}$. By (3) , condition $\operatorname{int}_{\gamma_{\mathcal{P}}}\left(\operatorname{cl}_{\gamma_{\mathcal{P}}}(A)\right)=\emptyset$ is equivalent to $\operatorname{int}_{\gamma}\left(\operatorname{cl}_{\gamma}\left(\tilde{A}_{n_{0}}\right)\right)=\emptyset$, which gives (4).

In the consecutive step of the proof, we will show that

if $A \subset X \times\left\{n_{0}\right\}$ for some $n_{0} \in \mathbb{N}$ and $\tilde{A}_{n_{0}}$ is of second category in $(X, \gamma)$ then $A$ is of second category in $\left(X \times \mathbb{N}, \gamma_{\mathcal{P}}\right)$.

Suppose, contrary to our claim, that $A$ is a meager set in $\left(X \times \mathbb{N}, \gamma_{\mathcal{P}}\right)$. Then $A=\bigcup_{k \in \mathbb{N}} B^{k}$, where $B^{k} \subset X \times\left\{n_{0}\right\}$, and $B^{k}$ is $\gamma_{\mathcal{P}}$-nowhere dense for each $k \in \mathbb{N}$. According to (4) $\tilde{B}_{n_{0}}^{k}$ is $\gamma$-nowhere dense, so $\tilde{A}_{n_{0}}=\bigcup_{k \in \mathbb{N}} \tilde{B}_{n_{0}}^{k}$ is a meager set in $(X, \gamma)$, which is impossible.

We are able now to prove that $\left(X \times \mathbb{N}, \gamma_{\mathcal{P}}\right)$ is a Baire space. Let $U \in \gamma_{\mathcal{P}} \backslash\{\emptyset\}$. We have $U=\bigcup_{n \in \mathbb{N}}\left(\tilde{U}_{n} \times\{n\}\right)$ and $\tilde{U}_{n} \in \gamma$ for $n \in \mathbb{N}$. Obviously, there exists $n_{1} \in \mathbb{N}$ such that $\tilde{U}_{n_{1}} \times\left\{n_{1}\right\} \neq \emptyset$. Since $(X, \gamma)$ is a Baire space, we have that $\tilde{U}_{n_{1}}$ is of second category in $(X, \gamma)$. By (5), $\tilde{U}_{n_{1}} \times\left\{n_{1}\right\}$ is of second category in $\left(X \times \mathbb{N}, \gamma_{\mathcal{P}}\right)$, which gives that $U$ is of second category in $\left(X \times \mathbb{N}, \gamma_{\mathcal{P}}\right)$. Thus, $\left(X \times \mathbb{N}, \gamma_{\mathcal{P}}\right)$ is a Baire space. 
Consider the space $\left(\mathbb{Q}, T_{\mathbb{Q}}\right)$. Obviously, it is not a Baire space. Let $\left\{q_{n}\right\}_{n \in \mathbb{N}}$ be a sequence of all rational numbers. Put $f(x)=q_{n}$ for $x \in X \times\{n\}, n \in \mathbb{N}$. Obviously $f: X \times \mathbb{N} \longrightarrow \mathbb{Q}$, and $f$ is a continuous surjection.

In the next theorem, we will formulate an assumption under which an image of Baire sGTS is Baire sGTS.

THEOREM 2.4. Let $\left(X, \gamma_{X}\right)$ and $\left(Y, \gamma_{Y}\right)$ be sGTSs. If a surjection $f: X \rightarrow Y$ is a q-semi-homeomorphism and $\left(X, \gamma_{X}\right)$ is a Baire space then $\left(Y, \gamma_{Y}\right)$ is a Baire space.

P r o o f. Let $\left\{W_{n}\right\}_{n \in \mathbb{N}}$ be a sequence of open and dense sets in $Y$. First, we show that $\left\{\operatorname{int}_{X}\left(f^{-1}\left(W_{n}\right)\right)\right\}_{n \in \mathbb{N}}$ is a sequence of open and dense sets in $X$.

Fix $n \in \mathbb{N}$. Notice that

the set $f^{-1}\left(W_{n}\right)$ is dense in $X$.

Indeed, let $U \in \gamma_{X} \backslash\{\emptyset\}$. Since $f$ is semi-open, we have $\emptyset \neq f(U) \subset \operatorname{cl}_{Y}\left(\operatorname{int}_{Y}(f(U))\right)$. Thus, $\operatorname{int}_{Y}(f(U)) \neq \emptyset$. Since $W_{n}$ is dense, we have $\operatorname{int}_{Y}(f(U)) \cap W_{n} \neq \emptyset$, so let $y_{0} \in f(U) \cap W_{n}$. There exists $x_{1} \in U$, such that $f\left(x_{1}\right)=y_{0} \in W_{n}$. Hence, we have $x_{1} \in U \cap f^{-1}\left(W_{n}\right)$, and consequently, $U \cap f^{-1}\left(W_{n}\right) \neq \emptyset$. From arbitrariness of $U$, we obtain (6).

Now, we will prove that $\operatorname{int}_{X}\left(f^{-1}\left(W_{n}\right)\right)$ is dense in $X$. Since $f$ is semi-continuous, we obtain that $f^{-1}\left(W_{n}\right)$ is semi-open, so $f^{-1}\left(W_{n}\right) \subset \operatorname{cl}_{X}\left(\operatorname{int}_{X}\left(f^{-1}\left(W_{n}\right)\right)\right)$. Hence, and from (6), we have $X=\operatorname{cl}_{X}\left(\operatorname{int}_{X}\left(f^{-1}\left(W_{n}\right)\right)\right)$. Thus, $\operatorname{int}_{X}\left(f^{-1}\left(W_{n}\right)\right)$ is dense in $X$.

Since $\left(X, \gamma_{X}\right)$ is a Baire space, by Theorem 2.2, we have that

$$
T=\bigcap_{n \in \mathbb{N}} \operatorname{int}_{X}\left(f^{-1}\left(W_{n}\right)\right) \text { is dense in } X .
$$

We will show that

$$
f(T) \text { is dense in } Y \text {. }
$$

Let $W \in \gamma_{Y} \backslash\{\emptyset\}$. Since $f$ is a surjection and semi-continuous, so $\emptyset \neq f^{-1}(W) \subset$ $\operatorname{cl}_{X}\left(\operatorname{int}_{X}\left(f^{-1}(W)\right)\right) \cdot\left(X, \gamma_{X}\right)$ is sGTS, so $\operatorname{int}_{X}\left(f^{-1}(W)\right) \neq \emptyset$. Since $T$ is dense in $X$, so $\operatorname{int}_{X}\left(f^{-1}(W)\right) \cap T \neq \emptyset$. That clearly means that $f^{-1}(W) \cap T \neq \emptyset$, so there exists $x_{0} \in f^{-1}(W) \cap T$. Thus, $f\left(x_{0}\right) \in W \cap f(T)$, so $W \cap f(T) \neq \emptyset$. From arbitrariness of $W$, we obtain (77).

Moreover, we have $f(T) \subset \bigcap_{n \in \mathbb{N}} W_{n}$, so by (7),$\bigcap_{n \in \mathbb{N}} W_{n}$ is dense.

By Theorem 2.2 we have that $\left(Y, \gamma_{Y}\right)$ is a Baire space.

In [7], Á. Császár noticed that, having sGTS $(X, \gamma)$, one can consider new sGTS $(X, \sigma(\gamma))$. In particular, if a classical topological space $(X, \mathcal{T})$ will be a starting point, then one can consider a derived $\operatorname{GTS}(X, \sigma(\mathcal{T}))$. The natural 


\section{ON SEMI-OPEN SETS OF FUNCTIONS}

question arises whether an $(X, \sigma(\mathcal{T}))$ is a Baire GTS whenever $(X, \mathcal{T})$ is a Baire space. In the case of GTS, the situation is more complicated because different kinds of nowhere density lead to distinguishing at least three kinds of Baire GTSs ([18]). However, in many considerations, weak Baire spaces are the most important.

LEMMA 2.5. Let $(X, \mathcal{T})$ be a topological space, and $K \subset X$. The set $K$ is $\mathcal{T}$-nowhere dense if and only if $K$ is $\sigma(\mathcal{T})$-strongly nowhere dense.

P r o o f. Necessity. Let $U \in \sigma(\mathcal{T}) \backslash\{\emptyset\}$. Then $U \subset \operatorname{cl}_{\mathcal{T}}\left(\operatorname{int}_{\mathcal{T}}(U)\right)$, so $\operatorname{int}_{\mathcal{T}}(U) \neq \emptyset$. Since $K$ is $\mathcal{T}$-nowhere dense, there exists a nonempty set $V \in \mathcal{T} \subset \sigma(\mathcal{T})$ such that $V \subset \operatorname{int}_{\mathcal{T}}(U) \subset U$ and $V \cap K=\emptyset$. Thus, $K$ is $\sigma(\mathcal{T})$-strongly nowhere dense.

Sufficiency. Let $U \in \mathcal{T} \backslash\{\emptyset\}$. Clearly, $U \in \sigma(\mathcal{T})$. Since $K$ is $\sigma(\mathcal{T})$-strongly nowhere dense, there exists a nonempty set $V \in \sigma(\mathcal{T})$ such that $V \subset U$ and $V \cap K=\emptyset$. We have $\emptyset \neq V \subset \operatorname{cl}_{\mathcal{T}}\left(\operatorname{int}_{\mathcal{T}}(V)\right)$, so $\operatorname{int}_{\mathcal{T}}(V) \neq \emptyset$. Moreover, $\operatorname{int}_{\mathcal{T}}(V) \subset U$ and $\operatorname{int}_{\mathcal{T}}(V) \cap K=\emptyset$. Thus, $K$ is $\mathcal{T}$-nowhere dense.

ThEOREM 2.6. Let $(X, \mathcal{T})$ be a topological space. $(X, \mathcal{T})$ is a Baire space if and only if $(X, \sigma(\mathcal{T}))$ is a weak Baire space.

P r o of. Necessity. Suppose, contrary to our claim, that $(X, \sigma(\mathcal{T}))$ is not a weak Baire space. Then, there exists a nonempty set $U \in \sigma(\mathcal{T})$ such that $U=\bigcup_{n \in \mathbb{N}} K_{n}$, where $K_{n}$ are strongly nowhere dense sets in $(X, \sigma(\mathcal{T}))$. By Lemma 2.5, the sets $K_{n}$ are $\mathcal{T}$-nowhere dense. Moreover, $\emptyset \neq \operatorname{int}_{\mathcal{T}}(U) \subset \bigcup_{n \in \mathbb{N}} K_{n}$, so $\operatorname{int}_{\mathcal{T}}(U)$ is nonempty $\mathcal{T}$-open and $\mathcal{T}$-meager set, which contradicts the fact that $(X, \mathcal{T})$ is a Baire space.

Sufficiency. Suppose that $(X, \mathcal{T})$ is not a Baire space. Then, there exists a nonempty set $U \in \mathcal{T} \subset \sigma(\mathcal{T})$ such that $U=\bigcup_{n \in \mathbb{N}} K_{n}$, where $K_{n}$ are $\mathcal{T}$-nowhere dense. By Lemma 2.5 the sets $K_{n}$ are strongly nowhere dense in $(X, \sigma(\mathcal{T}))$. Thus, $U$ is nonempty $\sigma(\mathcal{T})$-s-meager set which belongs to $\sigma(\mathcal{T})$. This contradicts the fact that $(X, \sigma(\mathcal{T}))$ is a weak Baire space.

The fact of considering weak Baire GTS in the above theorem justifies the more detailed examining of these spaces. The possibility of combining considerations concerning topological spaces and generalized topological spaces suggests another interesting problem. The starting point is the known theorem saying that, in the case of topological spaces, the open subspace of a Baire space is a Baire space. The basic question is whether this relationship is also true in the case of weak Baire GTS. A simple corollary from the next theorem is a negative answer to this question. However, the theorem contains much stronger result.

THEOREM 2.7. For each topological space $(Y, \mathcal{T})$ there exists a weak Baire sGTS $(X, \gamma)$ such that $Y \in \gamma$ and $\mathcal{T}=\gamma \uparrow Y=\{A \cap Y: A \in \gamma\}$. 
P r o o f. First, discuss briefly a special case $\mathcal{T}=\{Y, \emptyset\}$. Then, $(Y, \mathcal{T})$ is a Baire space and therefore a weak Baire space. So, it is sufficient to put $X=Y$ and $\gamma=\mathcal{T}$

In other cases, we assume that $\operatorname{card}(\mathcal{T}) \geq 3$.

Now, consider the case $\operatorname{card}(\mathrm{Y}) \geq \aleph_{0}$. Denote $\operatorname{card}(\mathrm{Y})=\mathfrak{n}$ and $\operatorname{card}(\mathcal{T})=\mathfrak{m}$. Put $Z=Y \cup \mathcal{T}$ (notice that $Z$ contains elements of $Y$ as well as sets consisting of elements of $Y$ ). We will define the set $S$ taking into account the relation between the numbers $\mathfrak{n}$ and $\mathfrak{m}$.

If $\mathfrak{n} \leq \mathfrak{m}$, then put $S=\mathcal{T}$, and we have $\operatorname{card}(\mathrm{S})=\operatorname{card}(\mathcal{T})=\mathfrak{m} \geq \mathfrak{n}=\operatorname{card}(\mathrm{Y})$. If $\mathfrak{n}>\mathfrak{m}$, then put $S=Y$, and we have $\operatorname{card}(\mathrm{S})=\operatorname{card}(\mathrm{Y})=\mathfrak{n}>\mathfrak{m}=\operatorname{card}(\mathcal{T})$.

Thus, in both cases, the following inequalities are true: $\operatorname{card}(\mathrm{S}) \geq \operatorname{card}(\mathrm{Y})$, $\operatorname{card}(\mathrm{S}) \geq \operatorname{card}(\mathcal{T})$ and $\operatorname{card}(\mathrm{S}) \leq \operatorname{card}(\mathrm{Z})$.

Let $X_{2}=2^{Z} \backslash Z$. Then, $\operatorname{card}\left(\mathrm{X}_{2}\right) \geq \operatorname{card}(\mathrm{S})$ and $X_{2} \cap Z=\emptyset$.

Let $\left(x_{\xi}\right)_{\xi<\alpha}$ be a transfinite sequence consisting of all elements of the set $X_{2}$, where $\alpha$ is an ordinal number. Create a set $X_{1}=\left\{\left\{x_{\xi}, x_{\xi+1}\right\}: \xi<\alpha\right\}$ if $\alpha$ is a limit ordinal or $X_{1}=\left\{\left\{x_{\xi}, x_{\xi+1}\right\}: \xi<\alpha-1\right\} \cup\left\{\left\{x_{\alpha-1}, x_{1}\right\}\right\}$, if $\alpha$ is not a limit ordinal. Clearly, $\operatorname{card}\left(\mathrm{X}_{1}\right)=\operatorname{card}\left(\mathrm{X}_{2}\right) \geq \operatorname{card}(\mathrm{S}) \geq \operatorname{card}(\mathcal{T})$. To $\operatorname{simplify}$ further notation, we assume that if $\alpha$ is not a limit ordinal, writing $\left\{x_{\xi}, x_{\xi+1}\right\}$ for $\xi=\alpha-1$, we will mean $\left\{x_{\alpha-1}, x_{1}\right\}$. Let $X_{0} \subset X_{1}$ be a set such that $\operatorname{card}\left(\mathrm{X}_{0}\right)=\operatorname{card}(\mathcal{T})$. Let $\zeta: \mathcal{T} \rightarrow X_{0}$ be an injection. Adopt the following notation: if $U \in \mathcal{T}$, then $\xi_{U}$ will denote an ordinal number less than $\alpha$, such that $\zeta(U)=\left\{x_{\xi_{U}}, x_{\xi_{U}+1}\right\}$.

Put $X=Y \cup \bigcup_{U \in \mathcal{T} \backslash\{Y\}}\left\{x_{\xi_{U}}, x_{\xi_{U}+1}\right\}$. Let $\mathcal{P}=\{Y, \emptyset\} \cup\left\{U \cup\left\{x_{\xi_{U}}, x_{\xi_{U}+1}\right\}\right.$ : $U \in \mathcal{T} \backslash\{Y\}\}$. It is easy to see that $\left(X, \gamma_{\mathcal{P}}\right)$ is $\operatorname{sGTS}$ and $\gamma_{\mathcal{P}} \uparrow Y=\mathcal{T}$.

What is left to do is to show that $\left(X, \gamma_{\mathcal{P}}\right)$ is a weak Baire space. It is sufficient to prove that there are no nonempty strongly nowhere dense sets in the space $\left(X, \gamma_{\mathcal{P}}\right)$. In order to prove this fact, we will show that

$$
\text { no singleton is strongly nowhere dense. }
$$

Let $x \in X$ and consider the set $\{x\}$. Assume first that

$$
x \in \bigcup_{U \in \mathcal{T} \backslash\{Y\}}\left\{x_{\xi_{U}}, x_{\xi_{U}+1}\right\} .
$$

Then, there exists $U \in \mathcal{T} \backslash\{Y\}$ such that

$$
x \in\left\{x_{\xi_{U}}, x_{\xi_{U}+1}\right\} \subset U \cup\left\{x_{\xi_{U}}, x_{\xi_{U}+1}\right\} .
$$

From the facts that $\zeta$ is an injection and $X_{2} \cap Y=\emptyset$, one can easily conclude that the set $U \cup\left\{x_{\xi_{U}}, x_{\xi_{u}+1}\right\}$ does not contain any nonempty proper subset belonging to $\gamma_{\mathcal{P}}$. Now, assume that $x \in Y$. Since $X_{2} \cap Y=\emptyset$ and all the sets from $\gamma_{\mathcal{P}}$ except of $Y$ and $\emptyset$ have nonempty intersection with $X_{2}$, we conclude that $Y$ has no nonempty proper subset belonging to $\gamma_{\mathcal{P}}$. Thus, we obtain (8). 
Obviously, (8) implies that there are no nonempty strongly nowhere dense sets in $\left(X, \gamma_{\mathcal{P}}\right)$, so $\left(X, \gamma_{\mathcal{P}}\right)$ is a weak Baire space.

Now, we consider the case $\operatorname{card}(\mathrm{Y})<\aleph_{0}$. Clearly, $\operatorname{card}(\mathcal{T})<\aleph_{0}$. Put $\mathcal{T}=$ $\left\{U_{1}, \ldots, U_{k}\right\}$. Let $X_{0}$ be a set of real numbers $x_{1}, x_{2}, \ldots, x_{k}, x_{k+1}$ such that $x_{i} \notin Y$ for $i=1,2, \ldots, k+1$ and $x_{i} \neq x_{j}$ for $i \neq j$. Put $X=Y \cup X_{0}$. Let $\zeta: \mathcal{T} \rightarrow\left\{\left\{x_{i}, x_{i+1}\right\}: i=1,2, \ldots, k\right\}$ be a function defined as follows $\zeta\left(U_{i}\right)=\left\{x_{i}, x_{i+1}\right\}$ for $i=1, \ldots, k$. Let $\mathcal{P}=\{\emptyset, Y\} \cup\left\{U_{i} \cup\left\{x_{i}, x_{i+1}\right\}: U_{i} \neq Y\right\}$. It is easy to see that $\left(X, \gamma_{\mathcal{P}}\right)$ is sGTS and $\gamma_{\mathcal{P}} \uparrow Y=\mathcal{T}$.

Similar to the case $\operatorname{card}(\mathrm{Y}) \geq \aleph_{0}$, one can show that $\left(X, \gamma_{\mathcal{P}}\right)$ is a weak Baire space.

\section{Transitivity in the primal and generated topological structures}

The terminology in the area of transitive and nomadic functions is not unified (see [2], [5], [15], 22], [24, [26], 27]). Therefore, we will start the considerations in this section with adopting the following definitions.

Let $(X, \gamma)$ be sGTS and $f:(X, \gamma) \rightarrow(X, \gamma)$. We say that $f$ is a transitive function if it fulfils the following condition

for every pair of nonempty $\gamma$-open sets $U$ and $V$

there is a positive integer $n$ such that $f^{n}(U) \cap V \neq \emptyset$.

A function $f$ is called a nomadic function if

there is a $\gamma$-dense set $A \subset X$ such that

for each $x \in A$ the orbit $\Theta_{f}(x)$ is $\gamma$-dense.

If a function $f$ fulfils the condition

there is a point $x_{0} \in X$ such that the orbit $\Theta_{f}\left(x_{0}\right)$ is $\gamma$-dense,

then we call it a weakly nomadic function.

It is not difficult to notice

\section{Remark 3.1.}

(a) Every nomadic function is a weakly nomadic function.

(b) Every nomadic function is a transitive function.

However, even in topological spaces, conditions (T), (DDO), (DO) are not equivalent ([17, [21]). 
It occurs that in searching for conditions allowing to obtain equivalence of the above notions, a particular role may be played by the following condition imposed on the space $(X, \gamma)$ :

for each nonempty set $U \in \gamma$ and each $\left\{x_{1}, \ldots, x_{n}\right\} \subset X$

$$
\text { we have } \operatorname{int}_{\gamma}\left(U \backslash\left\{x_{1}, \ldots, x_{n}\right\}\right) \neq \emptyset \text {. }
$$

Obviously, there exists sGTS, which does not have property (D)

It is easy to notice that if $(X, \gamma)$ has property $(\mathrm{D})$ then the notions of weakly nomadic function and nomadic function become equivalent.

In the previous section of the paper, we have considered interesting transition from $\operatorname{GTS}(X, \gamma)$ to GTS $(X, \sigma(\gamma))$, in particular, transition from a topological space $(X, \mathcal{T})$ to generated by its $\operatorname{GTS}(X, \sigma(\mathcal{T}))$. From the point of view of the considerations led in this paper, it seems to be interesting to study the problem of equivalence of transitivity, nomadic property and weakly nomadic property as well as possibility of mutual transition of these properties for the same function considered in $(X, \mathcal{T})$ and $(X, \sigma(\mathcal{T}))$. The results concerning this problem are presented in Theorem 3.3 and Remark 3.4 .

We start our considerations with the following

Proposition 3.2. If $s G T S(X, \gamma)$ has property $(\mathrm{D})$ then $(X, \sigma(\gamma))$ has property (D)

P r o o f. Let $W \in \sigma(\gamma) \backslash\{\emptyset\}$. Then, $W \subset \operatorname{cl}_{\gamma}\left(\operatorname{int}_{\gamma}(W)\right)$. Fix points $x_{1}, x_{2}, \ldots, x_{m}$. We will show that

$$
\text { there exists } U \in \sigma(\gamma) \backslash\{\emptyset\} \text { such that } U \subset W \backslash\left\{x_{1}, x_{2}, \ldots, x_{m}\right\} \text {. }
$$

Put $U=\operatorname{int}_{\gamma}\left(W \backslash\left\{x_{1}, x_{2}, \ldots, x_{m}\right\}\right)$. We have $U \subset \operatorname{cl}_{\gamma}\left(\operatorname{int}_{\gamma}\left(W \backslash\left\{x_{1}, x_{2}, \ldots, x_{m}\right\}\right)\right)=$ $\operatorname{cl}_{\gamma}\left(\operatorname{int}_{\gamma}\left(\operatorname{int}_{\gamma}\left(W \backslash\left\{x_{1}, x_{2}, \ldots, x_{m}\right\}\right)\right)\right)=\operatorname{cl}_{\gamma}\left(\operatorname{int}_{\gamma}(U)\right)$, so $U \in \sigma(\gamma)$.

All that is left is to show that $U \neq \emptyset$. Obviously, $\operatorname{int}_{\gamma}(W) \neq \emptyset$, so using the assumption that $(X, \gamma)$ has property (D) we conclude that there exists $B \in \gamma \backslash\{\emptyset\}$ such that $B \subset \operatorname{int}_{\gamma}(W) \backslash\left\{x_{1}, x_{2}, \ldots, x_{m}\right\} \subset W \backslash\left\{x_{1}, x_{2}, \ldots, x_{m}\right\}$. Therefore, $U=\operatorname{int}_{\gamma}\left(W \backslash\left\{x_{1}, x_{2}, \ldots, x_{m}\right\}\right) \neq \emptyset$.

We now turn to our main problem.

First, we pay the reader's attention to the fact that the proofs of Theorems 3.3 and 3.8 are close to the proofs of analogous theorems considered also in the context of GTS (e.g., 20]). However, in these theorems, we aimed to obtain as weak assumptions as possible which led to concerning (D) property in Theorem 3.3 and (D) and (TOP) properties in Theorem 3.8. Therefore, even analogous considerations contain subtle differences. For that reason, we present these proofs in their entirety. 
THEOREM 3.3. Let $(X, \gamma)$ be sGTS with property $(\mathrm{D})$ and such that $(X, \sigma(\gamma))$ is a Baire space with a countable base. Let $f:(X, \gamma) \rightarrow(X, \gamma)$ be irresolute. Then, the following conditions are equivalent:

(i) $f:(X, \gamma) \rightarrow(X, \gamma)$ is transitive;

(ii) $f:(X, \gamma) \rightarrow(X, \gamma)$ is nomadic;

(iii) $f:(X, \gamma) \rightarrow(X, \gamma)$ is weakly nomadic;

(iv) $f:(X, \sigma(\gamma)) \rightarrow(X, \sigma(\gamma))$ is transitive;

(v) $f:(X, \sigma(\gamma)) \rightarrow(X, \sigma(\gamma))$ is nomadic;

(vi) $f:(X, \sigma(\gamma)) \rightarrow(X, \sigma(\gamma))$ is weakly nomadic.

P r o of. The schema of the proof is as follows: (i) $\Rightarrow$ (iv) $\Rightarrow(\mathrm{v}) \Rightarrow$ (ii) $\Rightarrow$ (iii) $\Rightarrow$ (vi) $\Rightarrow(\mathrm{i})$.

(i) $\Rightarrow$ (iv) Let $U, V \in \sigma(\gamma) \backslash\{\emptyset\}$. Then, $U \subset \operatorname{cl}_{\gamma}\left(\operatorname{int}_{\gamma}(U)\right)$, and $V \subset \operatorname{cl}_{\gamma}\left(\operatorname{int}_{\gamma}(V)\right)$. Clearly, $\operatorname{int}_{\gamma}(U) \neq \emptyset \neq \operatorname{int}_{\gamma}(V)$, which follows from the fact that $(X, \gamma)$ is sGTS. By (i), there exists $k$ such that $f^{k}\left(\operatorname{int}_{\gamma}(U)\right) \cap \operatorname{int}_{\gamma}(V) \neq \emptyset$, and hence, $f^{k}(U) \cap$ $V \neq \emptyset$, which gives (iv).

(iv) $\Rightarrow(\mathrm{v})$ Notice first that the assumption of $f:(X, \gamma) \rightarrow(X, \gamma)$ being irresolute implies that $f:(X, \sigma(\gamma)) \rightarrow(X, \sigma(\gamma))$ is continuous.

Let $\left\{V_{1}, V_{2}, \ldots\right\}$ be a base of the space $(X, \sigma(\gamma))$ consisting of nonempty sets. For $i \in \mathbb{N}$ put $W_{i}=\bigcup_{n=0}^{\infty} f^{-n}\left(V_{i}\right)$. From continuity of $f:(X, \sigma(\gamma)) \rightarrow(X, \sigma(\gamma))$ and from the fact that $(X, \sigma(\gamma))$ is GTS, we conclude that for any $i \in \mathbb{N}$ we have $W_{i} \in \sigma(\gamma)$. Fix $i_{0} \in \mathbb{N}$. We will show that the set $W_{i_{0}}$ is $\sigma(\gamma)$-dense. Let $U \in \sigma(\gamma) \backslash\{\emptyset\}$. By (iv), there exists $k_{i_{0}} \in \mathbb{N}$ such that $f^{k_{i_{0}}}(U) \cap V_{i_{0}} \neq \emptyset$. Hence, $f^{-k_{i_{0}}}\left(V_{i_{0}}\right) \cap U \neq \emptyset$, so $W_{i_{0}} \cap U \neq \emptyset$, and thus the set $W_{i_{0}}$ is $\sigma(\gamma)$-dense. By Theorem 2.2 the set $\bigcap_{i \in \mathbb{N}} W_{i}$ is $\sigma(\gamma)$-dense.

Now, consider a point $x \in \bigcap_{i \in \mathbb{N}} W_{i}$. We will show that the orbit of $x$ is $\sigma(\gamma)$-dense. Let $U \in \sigma(\gamma)$ and $U \neq \emptyset$. There exists $i_{1} \in \mathbb{N}$ such that $V_{i_{1}} \subset U$. Obviously, $x \in W_{i_{1}}$ and there exists $n_{0} \in \mathbb{N} \cup\{0\}$ such that $x \in f^{-n_{0}}\left(V_{i_{1}}\right)$, so $f^{n_{0}}(x) \in V_{i_{1}} \subset U$. We have shown that each point of $\sigma(\gamma)$-dense set $\bigcap_{i \in \mathbb{N}} W_{i}$ has a $\sigma(\gamma)$-dense orbit.

(v) $\Rightarrow$ (ii) Let $W \in \gamma \backslash\{\emptyset\}$. We will show that the set $W$ contains a point with a $\gamma$-dense orbit. Clearly, $W \in \sigma(\gamma)$. By $(\mathrm{v})$, there exists a point $x_{W} \in$ $W$ such that $\Theta_{f}\left(x_{W}\right)$ is $\sigma(\gamma)$-dense. We will show that $\Theta_{f}\left(x_{W}\right)$ is $\gamma$-dense. Let $V \in \gamma \backslash\{\emptyset\} \subset \sigma(\gamma)$. Then, $V \cap \Theta_{f}\left(x_{W}\right) \neq \emptyset$. To finish the proof of this implication, it is sufficient to notice that the set $\left\{x_{W}: W \in \gamma \backslash\{\emptyset\}\right\}$ is $\gamma$-dense.

(ii) $\Rightarrow$ (iii) This implication is formulated above as Remark 3.1 (a).

(iii) $\Rightarrow$ (vi) Let $x_{0} \in X$ be such a point that the orbit $\Theta_{f}\left(x_{0}\right)$ is $\gamma$-dense. We will show that $\Theta_{f}\left(x_{0}\right)$ is $\sigma(\gamma)$-dense. Let $W \in \sigma(\gamma) \backslash\{\emptyset\}$. Then, $W \subset \operatorname{cl}_{\gamma}\left(\operatorname{int}_{\gamma}(W)\right)$. Obviously, $\operatorname{int}_{\gamma}(W) \in \gamma \backslash\{\emptyset\}$. Since $\Theta_{f}\left(x_{0}\right)$ is $\gamma$-dense, there exists $k \in \mathbb{N}$ such that $f^{k}\left(x_{0}\right) \in \operatorname{int}_{\gamma}(W) \subset W$. 
(vi) $\Rightarrow$ (i) Let $x_{0} \in X$ be a point with $\sigma(\gamma)$-dense orbit $\Theta_{f}\left(x_{0}\right)$. Since $\gamma \subset \sigma(\gamma)$, $\Theta_{f}\left(x_{0}\right)$ is $\gamma$-dense. Let $U, V \in \gamma \backslash\{\emptyset\}$. Then, the sets $U$ and $V$ contain points of the orbit $\Theta_{f}\left(x_{0}\right)$. Let $m_{0}=\min \left\{m \in \mathbb{N} \cup\{0\}: f^{m}\left(x_{0}\right) \in U\right\}$ and $n_{0}=$ $\min \left\{n \in \mathbb{N} \cup\{0\}: f^{n}\left(x_{0}\right) \in V\right\}$.

Assume first that $m_{0}<n_{0}$ and put $k_{0}=n_{0}-m_{0}$. Then, $f^{k_{0}}\left(f^{m_{0}}\left(x_{0}\right)\right)=$ $f^{n_{0}}\left(x_{0}\right) \in V$, so $f^{k_{0}}(U) \cap V \neq \emptyset$.

Now, assume $m_{0} \geq n_{0}$ and put $L=\left\{i \in \mathbb{N} \cup\{0\}: n_{0} \leq i \leq m_{0} \wedge f^{i}\left(x_{0}\right) \in V\right\}$. Clearly, $L$ is nonempty finite set. By property (D) there exists a nonempty set $V^{*} \in \gamma$ such that $V^{*} \subset V \backslash\left\{f^{i}\left(x_{0}\right): i \in L\right\}$. Since $\Theta_{f}\left(x_{0}\right)$ is $\gamma$-dense, there exists $q \in \mathbb{N} \cup\{0\}$ such that $f^{q}\left(x_{0}\right) \in V^{*} \subset V$. We have $q>m_{0}$. Indeed, if $q \leq m_{0}$, then $q \in L$, which contradicts the fact that $f^{q}\left(x_{0}\right) \in V^{*}$. Put $t=q-m_{0}$. Then, $f^{t}\left(f^{m_{0}}\left(x_{0}\right)\right)=f^{q}\left(x_{0}\right) \in V$, so $f^{t}(U) \cap V \neq \emptyset$.

This completes the proof that $f:(X, \gamma) \rightarrow(X, \gamma)$ is transitive.

The authors of the paper have not established yet whether the assumption of the Baire property in the above theorem could be imposed on the space $(X, \gamma)$ instead of $(X, \sigma(\gamma))$.

Remark 3.4. Obviously, in Theorem 3.3. we can consider a topological space instead of sGTS.

In view of the earlier considerations, the converse situation seems to be interesting, i.e., we have a fixed $\operatorname{sGTS}(X, \gamma)$ and a topological space $(X, \mathcal{T}(\gamma))$ generated by $(X, \gamma)([9])$, where $\mathcal{T}(\gamma)=\left\{A \subset X: \forall_{B \in \gamma} A \cap B \in \gamma\right\}$. Is it possible to prove a theorem analogous to Theorem 3.3. We will start our considerations with formulating two easily seen but useful lemmas and with an example showing some difficulty in the study of this issue.

LEMMA 3.5. Let $(X, \gamma)$ be sGTS. If $A \subset X$ is $\gamma$-dense, then $A$ is $\mathcal{T}(\gamma)$ -dense.

Let $(X, \gamma)$ be sGTS. One can easily conclude that if a function is $\gamma$-transitive, then it is $\mathcal{T}(\gamma)$-transitive. The converse implication is not true, which may be illustrated by the following example.

Example 3.6. Let $\mathcal{T}_{[0,1]}=\left\{A \cap[0,1]: A \in T_{N}\right\}$, where $T_{N}$ is the natural topology in $\mathbb{R}$. Put $X=[-1,1] \times[0,1], Y=[-1,0] \times[0,1], Z=[0,1] \times[0,1]$, $\gamma_{Y}=\left\{Y \backslash A: \operatorname{card}(\mathrm{A})<\aleph_{0}\right\} \cup\{\emptyset\}, \gamma_{Z}=\left\{U \times[0,1]: U \in \mathcal{T}_{[0,1]}\right\} \cup\{\emptyset\}$ and $\gamma=\left\{A \cup B: A \in \gamma_{Y}\right.$ and $\left.B \in \gamma_{Z}\right\}$. Then, $(X, \gamma)$ is sGTS with property (D). Let $\varphi:[0,1] \rightarrow[0,1]$ be given by the formula $\varphi(x)=1-|2 x-1|$ and consider the map $\psi: X \rightarrow X$ defined in the following way: $\psi(x)=(0,0)$ for $x \in Y$ and $\psi(x)=\left(\varphi\left(x_{1}\right), 0\right)$ for $x=\left(x_{1}, x_{2}\right) \in Z$. Then, $\psi:(X, \mathcal{T}(\gamma)) \rightarrow(X, \mathcal{T}(\gamma))$ is transitive but $\psi:(X, \gamma) \rightarrow(X, \gamma)$ is not transitive. 


\section{ON SEMI-OPEN SETS OF FUNCTIONS}

It occurs that if we impose an additional condition on the space $(X, \gamma)$ (further called property (TOP)), we obtain equivalence of $\gamma$-transitivity and $\mathcal{T}(\gamma)$-transitivity, which will be shown in Theorem 3.8

We will say that $\operatorname{sGTS}(X, \gamma)$ has property (TOP), if:

for each nonempty set $U \in \gamma$ there exists a nonempty set

$$
V \in \mathcal{T}(\gamma) \text { such that } V \subset U .
$$

Notice that $\operatorname{sGTS}(X, \gamma)$ in Example 3.6 does not have property (TOP).

Let us note one more theorem important for our considerations.

THEOREM 3.7. If sGTS $(X, \gamma)$ is a weak Baire space and has property (TOP), then $(X, \mathcal{T}(\gamma))$ is a Baire space.

P r o of. Suppose, contrary to our claim, that $(X, \mathcal{T}(\gamma))$ is not a Baire space. There exists a nonempty set $U \in \mathcal{T}(\gamma)$ such that $U=\bigcup_{n \in \mathbb{N}} K_{n}$, where $K_{n}$ are $\mathcal{T}(\gamma)$-nowhere dense. We have $U \in \gamma$. We will show that the sets $K_{n}$ are $\gamma$-strongly nowhere dense. Let $W \in \gamma \backslash\{\emptyset\}$. By property (TOP), there exists a nonempty set $W_{0} \in \mathcal{T}(\gamma)$ such that $W_{0} \subset W$. Since $K_{n}$ are $\mathcal{T}(\gamma)$-nowhere dense, there exists a nonempty set $V \in \mathcal{T}(\gamma)$ such that $V \subset W_{0}$ and $V \cap K_{n}=\emptyset$. Thus the sets $K_{n}$ are $\gamma$-strongly nowhere dense. We have shown that nonempty set $U \in \gamma$ is $\gamma$-s-meager, which contradicts the assumption that $(X, \gamma)$ is a weak Baire space.

TheOREM 3.8. Let $(X, \gamma)$ be a Baire sGTS with properties (TOP) and $(D)$ and such that the space $(X, \mathcal{T}(\gamma))$ has a countable base. Let $f:(X, \gamma) \rightarrow(X, \mathcal{T}(\gamma))$ be a continuous function. Then, the following conditions are equivalent:

(i) $f:(X, \gamma) \rightarrow(X, \gamma)$ is transitive;

(ii) $f:(X, \gamma) \rightarrow(X, \gamma)$ is nomadic;

(iii) $f:(X, \gamma) \rightarrow(X, \gamma)$ is weakly nomadic;

(iv) $f:(X, \mathcal{T}(\gamma)) \rightarrow(X, \mathcal{T}(\gamma))$ is tranisitive;

(v) $f:(X, \mathcal{T}(\gamma)) \rightarrow(X, \mathcal{T}(\gamma))$ is nomadic;

(vi) $f:(X, \mathcal{T}(\gamma)) \rightarrow(X, \mathcal{T}(\gamma))$ is weakly nomadic.

P r o of. The schema of the proof is as follows: (iii) $\Rightarrow$ (vi) $\Rightarrow$ (v) $\Rightarrow$ (iv) $\Rightarrow$ (i) $\Rightarrow$ (ii) $\Rightarrow$ (iii).

(iii) $\Rightarrow$ (vi) It follows from Lemma 3.5 .

(vi) $\Rightarrow(\mathrm{v})$ Let $x_{0} \in X$ be such that its orbit $\Theta_{f}\left(x_{0}\right)$ is $\mathcal{T}(\gamma)$-dense. We will show that

for any $i>0$ the point $f^{i}\left(x_{0}\right)$ has $\mathcal{T}(\gamma)$-dense orbit.

Fix $i_{0}>0$. We have $\Theta_{f}\left(f^{i_{0}}\left(x_{0}\right)\right)=\left\{f^{i_{0}}\left(x_{0}\right), f^{i_{0}+1}\left(x_{0}\right), f^{i_{0}+2}\left(x_{0}\right), \ldots\right\}$. Let $U \in \mathcal{T}(\gamma) \backslash\{\emptyset\}$. We have $U \in \gamma \backslash\{\emptyset\}$. Consider the set $U \backslash\left\{x_{0}, f\left(x_{0}\right), \ldots, f^{i_{0}-1}\left(x_{0}\right)\right\}$. 
By property (D) of the space $(X, \gamma)$, there exists a nonempty set $U_{0} \in \gamma$ such that $U_{0} \subset U \backslash\left\{x_{0}, \ldots, f^{i_{0}-1}\left(x_{0}\right)\right\} \subset U$. Property (TOP) of the space $(X, \gamma)$ implies that there exists $V_{0} \in \mathcal{T}(\gamma) \backslash\{\emptyset\}$ such that $V_{0} \subset U_{0}$. Since $\Theta_{f}\left(x_{0}\right)$ is $\mathcal{T}(\gamma)$-dense, there exists $k \in \mathbb{N}$ such that $f^{k}\left(x_{0}\right) \in V_{0} \subset U_{0} \subset U$. Obviously $k \geq i_{0}$, because for $j \in\left\{0, \ldots, i_{0}-1\right\}$, we have $f^{j}\left(x_{0}\right) \notin U_{0}$. Thus $f^{k}\left(x_{0}\right) \in \Theta_{f}\left(f^{i_{0}}\left(x_{0}\right)\right)$, so $\emptyset \neq V_{0} \cap \Theta_{f}\left(f^{i_{0}}\left(x_{0}\right)\right) \subset U \cap \Theta_{f}\left(f^{i_{0}}\left(x_{0}\right)\right)$. We have shown that, for any $i>0$, the orbit $\Theta_{f}\left(f^{i}\left(x_{0}\right)\right)$ is $\mathcal{T}(\gamma)$-dense. Clearly, the set $\left\{f^{i}\left(x_{0}\right): i>0\right\}$ is $\mathcal{T}(\gamma)$ -dense.

(v) $\Rightarrow$ (iv) Let $x_{0} \in X$ be a point with $\mathcal{T}(\gamma)$-dense orbit $\Theta_{f}\left(x_{0}\right)$. We will show that $f$ is $\mathcal{T}(\gamma)$-transitive. Let $U, V \in \mathcal{T}(\gamma) \backslash\{\emptyset\}$. Then, the sets $U$ and $V$ contain points of the orbit $\Theta_{f}\left(x_{0}\right)$. Let $m_{0}=\min \left\{m \in \mathbb{N} \cup\{0\}: f^{m}\left(x_{0}\right) \in U\right\}$ and $n_{0}=\min \left\{n \in \mathbb{N} \cup\{0\}: f^{n}\left(x_{0}\right) \in V\right\}$.

Assume first that $m_{0}<n_{0}$ and put $k_{0}=n_{0}-m_{0}$. Then

$$
f^{k_{0}}\left(f^{m_{0}}\left(x_{0}\right)\right)=f^{n_{0}}\left(x_{0}\right) \in V, \quad \text { so } \quad f^{k_{0}}(U) \cap V \neq \emptyset .
$$

Now, let $m_{0} \geq n_{0}$ and put $L=\left\{i \in \mathbb{N} \cup\{0\}: n_{0} \leq i \leq m_{0} \wedge f^{i}\left(x_{0}\right) \in V\right\}$. Obviously, $L$ is a nonempty finite set. By property (D) of the space $(X, \gamma)$, there exists a nonempty set $V^{*} \in \gamma$ such that $V^{*} \subset V \backslash\left\{f^{2}\left(x_{0}\right): i \in L\right\}$. According to property (TOP) of $(X, \gamma)$, there exists a nonempty set $V_{0} \in \mathcal{T}(\gamma)$ such that $V_{0} \subset V^{*}$. Since $\Theta_{f}\left(x_{0}\right)$ is $\mathcal{T}(\gamma)$-dense, there exists $q \in \mathbb{N}$ such that $f^{q}\left(x_{0}\right) \in V_{0} \subset$ $V^{*} \subset V$. We have $q>m_{0}$. Indeed, if $q \leq m_{0}$, then $q \in L$, which contradicts the fact that $f^{q}\left(x_{0}\right) \in V^{*}$. Put $t=q-m_{0}$. Then $f^{t}\left(f^{m_{0}}\left(x_{0}\right)\right)=f^{q}\left(x_{0}\right) \in V$, so $f^{t}(U) \cap V \neq \emptyset$, and the proof of the fact that $f$ is $\mathcal{T}(\gamma)$-transitive is finished.

(iv) $\Rightarrow$ (i) Assume $f$ is $\mathcal{T}(\gamma)$-transitive. Let $U, V \in \gamma \backslash\{\emptyset\}$. By property (TOP) of $(X, \gamma)$, there exist sets $U_{1}, V_{1} \in \mathcal{T}(\gamma) \backslash\{\emptyset\}$ such that $U_{1} \subset U$ i $V_{1} \subset V$. Since $f$ is $\mathcal{T}(\gamma)$-transitive, there exists $k \in \mathbb{N}$ such that $V_{1} \cap f^{k}\left(U_{1}\right) \neq \emptyset$, and we have $V \cap f^{k}(U) \neq \emptyset$, because $V_{1} \cap f^{k}\left(U_{1}\right) \subset V \cap f^{k}(U)$. Thus, $f$ is $\gamma$-transitive.

(i) $\Rightarrow$ (ii) Let $\left\{V_{1}, V_{2}, \ldots\right\}$ be a base of the space $(X, \mathcal{T}(\gamma))$ consisting of nonempty sets. For $i \in \mathbb{N}$, put $W_{i}=\bigcup_{n=0}^{\infty} f^{-n}\left(V_{i}\right)$. By continuity of $f:(X, \gamma) \rightarrow$ $(X, \mathcal{T}(\gamma))$ and by the fact that $\gamma$ is $\mathrm{GT}$, we conclude that $W_{i} \in \gamma$ for any $i \in \mathbb{N}$. Fix $i_{0} \in \mathbb{N}$. We will show that $W_{i_{0}}$ is $\gamma$-dense. Let $U \in \gamma \backslash\{\emptyset\}$. By condition (i), there exists $k_{i_{0}} \in \mathbb{N}$ such that $f^{k_{i_{0}}}(U) \cap V_{i_{0}} \neq \emptyset$. Hence $f^{-k_{i_{0}}}\left(V_{i_{0}}\right) \cap U \neq \emptyset$, and thus $W_{i_{0}} \cap U \neq \emptyset$. It means that the set $W_{i_{0}}$ is $\gamma$-dense. Since $i_{0}$ was chosen arbitrarily, the set $W_{i}$ is $\gamma$-dense for any $i \in \mathbb{N}$. According to Theorem 2.2 the set $\bigcap_{i \in \mathbb{N}} W_{i}$ is $\gamma$-dense.

Consider a point $x \in \bigcap_{i \in \mathbb{N}} W_{i}$. We will show that $x$ has a $\gamma$-dense orbit. Let $U \in \gamma$ and $U \neq \emptyset$. By (TOP), there exists a nonempty set $Z \in \mathcal{T}(\gamma)$ such that $Z \subset U$. Since $\left\{V_{1}, V_{2}, \ldots\right\}$ is a base of $(X, \mathcal{T}(\gamma))$, there existst $i_{1} \in \mathbb{N}$ such that $V_{i_{1}} \subset Z \subset U$. Obviously $x \in W_{i_{1}}$, so there exists $n_{0} \in \mathbb{N} \cup\{0\}$ such that $x \in f^{-n_{0}}\left(V_{i_{1}}\right)$, so $f^{n_{0}}(x) \in V_{i_{1}} \subset U$. We have shown that $\Theta_{f}(x)$ is $\gamma$-dense.

(ii) $\Rightarrow$ (iii) This implication is formulated above as Remark 3.1 (a). 


\section{ON SEMI-OPEN SETS OF FUNCTIONS}

\section{REFERENCES}

[1] ABDUL-JABBAR, A. M.: Topological property of $\theta$-semi-open sets, Int. J. Pure Appl. Sci. Technol. 13 (2012), 7-15.

[2] ALSEDA, L.-LLIBRE, J.-MISIUREWICZ, M.: Combinatorial Dynamics and Entropy in Dimension One. World Scientific Publ., Singapore, 1993.

[3] BAYHAN, S.-KANIBIR, A.-REILLY, I. L.: On functions between generalized topological spaces, Appl. Gen. Topol. 14 (2013), 195-203.

[4] BENCHALLI, S. S.-WALI, R. S.: On RW-closed sets in topological spaces, Bull. Malays. Math. Sci. Soc. (2) 30 (2007), 99-110.

[5] BRUCKNER, A. M.-HU, T.: On scrambled sets for chaotic functions, Trans. Amer. Math. Soc. 301 (1987), 289-297.

[6] CALDAS, M.-SARAF, R. K.: On approximately semiopen maps in topological spaces, Divulg. Mat. 14 (2006), 31-37.

[7] CSÁSZÁR, Á.: Generalized open sets, Acta Math. Hungar. 75 (1997), 65-87.

[8] CSÁSZĀR, Á.: Generalized topology, generalized continuity, Acta Math. Hungar. 96 (2002), 351-357.

[9] CSÁSZÁR, Á.: Generalized open sets in generalized topologies, Acta Math. Hungar. 106 (2005), 53-66.

[10] CSÁSZÁR, Á.: Weak structures, Acta Math. Hungar. 131 (2011), 193-195.

[11] EKICI, E.-JAFARI, S.: On a weaker form of complete irresoluteness, Bol. Soc. Paran. Mat. 26 (2008), 81-87.

[12] EKICI, E.: Further new generalized topologies via mixed constructions due to Császár, Math. Bohem. 140 (2015), 1-9.

[13] ENGELKING, R.: General Topology. Heldermann, Berlin, 1989.

[14] GENE CROSSLEY, S.-HILDEBRAND, S. K.: Semi-topological properties, Fund. Math. 74 (1972), 233-254.

[15] GotTschalK, W. H.-Hedlund, G. A.: Topological Dynamics, in: Amer. Math. Soc. Colloq. Publ., Vol. 36, Providence, RI, 1995.

[16] KALAIVANI, N.-SAI SUNDARA KRISHNAN, G.: On $\gamma$-generalized $\alpha$-continuous mappings in topological spaces, Bonfring Int. J. Data Mining 2 (2012), 23-27.

[17] KOLYADA, S.-SNOHA, L.: Some aspects of topological transitivity-a survey, Grazer Math. Ber. 334 (1997), 3-35.

[18] KORCZAK-KUBIAK, E.-LORANTY, A.-PAWLAK, R. J.: Baire generalized topological spaces, generalized metric spaces and infinite games, Acta Math. Hung. 140 (2013), 203-231.

[19] LI, J.: Generalized topologies generated by subbases Acta Math. Hung. 114 (2007), 1-12.

[20] LORANTY, A.-PAWLAK, R. J.: On the transitivity of multifunctions and density of orbits in generalized topological spaces, Acta Math. Hungar. 135 (2012), 56-66.

[21] MAI, J. H.-SUN, W. H.: Transitivities of maps of general topological spaces, Topology Appl. 157 (2010), 946-953.

[22] DE MELO, W.-VAN STRIEN, S.: One-Dimensional Dynamics. Springer, Berlin, 1993.

[23] MICHAEL, F. I.: On semi-open sets with respect to an ideal, Eur. J. Pure Appl. Math. 6 (2013), 53-58.

[24] PETERSEN, K.: Ergodic Theory, in: Cambridge Stud. Adv. Math., Vol. 2, Cambridge Univ. Press, Cambridge, 1983.

[25] POWAR, P. L.-RAJAK, K. L.: Some new concepts of continuity in generalized topological space, Int. J. Comput. Appl. 38 (2012), 12-17. 


\section{EWA KORCZAK-KUBIAK - RYSZARD J. PAWLAK}

[26] SIDOROV, Y. A.: Topologically indecomposable transformations of the n-dimensional space, Volzh. Mat. Sbornik 5 (1966), 326-330. (In Russian)

[27] WALTERS, P.: An Introduction to Ergodic Theory. Springer, New York, 1982.

Received November 12, 2015

Ewa Korczak-Kubiak

Ryszard J. Pawlak

Faculty of Mathematics and

Computer Science

Łódź University

Banacha 22

PL-90-238 É́dź

POLAND

E-mail: ekor@math.uni.lodz.pl rpawlak@math.uni.lodz.pl 